\title{
Large Thermal Expansivity of Clathrate Hydrates
}

\author{
Hideki Tanaka* and Yoshinori Tamai \\ Department of Polymer Chemistry, Graduate School of Engineering, Kyoto University, \\ Sakyo, Kyoto 606-01, Japan
}

\author{
Kenichiro Koga \\ Department of Chemistry, 572 Hamilton Hall, University of Nebraska-Lincoln, \\ Lincoln, Nebraska 68588 \\ Received: February 10, 1997; In Final Form: June 4, $1997^{\otimes}$
}

\begin{abstract}
The free energies of clathrates are calculated over a wide range of temperatures in order to explain a large thermal expansivity of clathrate hydrates compared with that of ice. Several proton-disordered configurations for hexagonal ice and type I chathrate hydrates are generated. The free energy is approximated to the sum of the minimum potential energy, the harmonic free energy, and the configurational entropy arising from the disordered nature of protons. The free energy at a given temperature is minimized with respect to the volume of the system. This enables us to evaluate the thermal expansivity from only intermolecular interaction potentials. The larger thermal expansivity of clathrate hydrates than ice is successfully reproduced. It is found that the large expansivity of clathrate hydrate structure I stems from the existence of guest molecules and that a difference in oxygen atom arrangement between clathrate hydrates and ice plays a minor role. The effective potential energy surface of a guest molecule becomes harmonic with an increase in temperature. This seems to undermine the large difference in the thermal expansivity between clathrate hydrates and ice.
\end{abstract}

\section{Introduction}

Gas hydrates contain guest molecules in the cavities made from host water molecules which are firmly hydrogen-bonded with each other. ${ }^{1,2}$ The clathrate hydrate is stable only when the interaction between guest and water molecules dominates over the sum of the two unfavorable terms: (1) entropy decrease arising from confinement of guest molecules in small void cages and (2) free energy for formation of empty clathrate hydrate structure from ice or liquid water. The thermodynamic stability of clathrate hydrates has long been accounted for by van der Waals and Platteeuw (vdWP) theory. ${ }^{3}$ Recently, it has been applied to various clathrate hydrates with a newly proposed method of calculating the free energy of cage occupation from intermolecular interactions. Thus, prediction of dissociation pressure was much improved without invoking empirical parameters. $^{4-6}$

It is not surprising that most thermodynamic and dynamic properties of clathrate hydrates are similar to those of ice since the interaction between guest and host water molecules is not so strong as to alter those properties originating mostly from the hydrogen bonds. However, the thermal expansivity and the thermal conductivity of clathrate hydrates are exceptionally different from those of ice. ${ }^{2}$ The thermal conductivity of clathrate hydrates is roughly $20 \%$ of ice near the ice point and is 1 order of magnitude smaller at low temperatures below 150 $\mathrm{K}^{7-10}$ The thermal conductivity of ice is inversely proportional to temperature, as is the case of the normal crystal. On the other hand, that of hydrate is proportional to temperature, although its temperature dependence is not so distinct. Molecular dynamics (MD) simulations have been performed to account for the anomalous conductivity and support the "resonance scattering model". ${ }^{11-14}$

* To whom all correspondence should be addressed. E-mail address: tanaka@ @uic.kyoto-u.ac.jp.

${ }^{\otimes}$ Abstract published in Advance ACS Abstracts, July 15, 1997.
The thermal expansivity of ethylene oxide clathrate hydrate is nearly twice as large as that of ice. ${ }^{15-17}$ This difference can be accounted for by a difference in either host water or guest molecules. The former is a difference in the arrangement of host water molecules; ice is made from hexagonal puckered rings, while the clathrate hydrate is composed of planar hexagonal and pentagonal rings. The latter is an effect of guest molecules: the coupling of guest and host water or the guest vibrations, or both. To clarify this point, a hypothetical clathrate hydrate should be examined, which contains no guest molecule but has the same molecular arrangement of water molecules as the real hydrate. It is also important to examine whether the potential energy or the vibrational free energy is the most crucial factor in the large difference.

Tse et al. performed MD simulation in which the large thermal expansivity was successfully reproduced. ${ }^{16}$ They concluded that the structure difference contributes little to the difference in the thermal expansivity between clathrate hydrates and ice. In order to gain further insight into the origin of the large thermal expansivity, it is highly desirable to calculate the free energy of clathrate hydrates as a function of both temperature and volume. This enables us to evaluate the various contributions to the total free energy, separately. The equilibrium configuration at a given temperature can be obtained by minimizing the free energy of the system with respect to volume. The free energy of ice and clathrate hydrate I is given by the sum of the minimum potential energy, the free energy of intermolecular vibrations, and the configurational entropy, the last of which arises from the proton-disordered nature of those crystallites. The configurational entropies per molecule for both ice and clathrate hydrate forms are given by $k \ln (3 / 2)$ with great accuracy, where $k$ is the Boltzmann constant. The configurational entropies are independent of temperature and density and therefore are ignored in the following calculations. Thus, the free energy is approximated to the sum of the potential energy 
at zero temperature and the harmonic free energy, thereby introducing explicit temperature and density dependence.

In the present study, the thermal expansivity is calculated, and the most likely mechanism of large thermal expansivity is delineated. The present paper is organized as follows. In section II, methods to calculate the thermal expansivity are described. In section III, results on the thermal expansivity are presented and a difference in the free energy between ice and clathrate hydrates is examined. Conclusions are given in section IV.

\section{Theory and Method}

a. Intermolecular Interaction and Structure of the Unit Cell. In the present study, all interactions are assumed to be pairwise additive. The water-water intermolecular interaction is described by the TIP4P potential. ${ }^{18}$ The $\mathrm{Xe}-\mathrm{Xe}$ interaction is given by a spherical Lennard-Jones (LJ) potential with the size and energy parameters of $4.047 \AA$ and $1.9205 \mathrm{~kJ} \mathrm{~mol}^{-1} \cdot{ }^{19}$ For the water-guest interaction, we assume the LorentzBerthelot rule with the LJ parameters for the oxygen atom on the water molecule set equal to those for TIP4P water: $\sigma_{\mathrm{OO}}=$ $3.154 \AA$ and $\epsilon_{\mathrm{OO}}=0.6487 \mathrm{~kJ} \mathrm{~mol}^{-1}$. The interaction potentials for all pairs of molecules are truncated smoothly at $8.655 \AA$ by multiplying a switching function. ${ }^{4}$

The unit cell of the hydrate $\mathrm{I}$ is cubic and the experimental lattice parameter is used in the following calculation; the size of the unit cell is $12.03 \AA .{ }^{1,2}$ There exist six large and two small cages in the unit cell of clathrate hydrate I. The number of water molecules is 368 , which corresponds to eight unit cells of type I clathrate hydrate, and the edge size of the cubic basic cell is $24.06 \AA$. The guest Xe atoms occupy all 64 available cages. The basic cell of low-pressure hexagonal ice (ice Ih) is orthorhombic, containing 288 water molecules. The size of the cell is $(a \times b \times c=) 23.67 \times 18.23 \times 22.33 \AA .^{20}$ Those cell parameters are not fixed but are adjusted so that the free energy of the system has a minimum value at a given temperature as described below.

Six configurations are generated for both clathrate hydrate I and hexagonal ice; all the structures are of proton-disordered form. The orientations of individual water molecules are so assigned as to satisfy the Bernal-Fowler rule with a vanishing value of the dipole moment but otherwise random. The potential energy of each system is minimized by a standard quenching method prior to the normal mode analysis.

b. Free Energy Calculation. In order to obtain the density of state, $g(\omega)$, for intermolecular vibrational motions, normal mode analysis is performed by diagonalizing the mass-weighted force constant matrix $\mathbf{m}^{-1 / 2} \mathbf{V} \mathbf{m}^{-1 / 2}$, where $\mathbf{m}$ is the appropriately defined mass tensor and $\mathbf{V}$ is calculated by direct differentiation of the intermolecular interaction. The density of state for intermolecular vibration is obtained by a simple average of six configurations generated. Once the density of state is obtained, the calculation of the harmonic free energy $f_{\mathrm{v}}$ is straightforward. The harmonic free energy per molecule at temperature $T$ is given via a quantum mechanical partition function for a harmonic oscillator as

$$
f_{\mathrm{v}}=k T \int \ln [2 \sinh (\hbar \omega / 2 k T)] g(\omega) \mathrm{d} \omega
$$

where $\hbar$ is the Planck constant divided by $2 \pi$. The total free energy is approximated to the sum of the interaction energy and the (intermolecular) vibrational free energy $f_{\mathrm{v}}$, neglecting both the configurational entropy and the $p V$ (pressure $\times$ volume) terms, the latter of which is negligibly small at atmospheric pressure. That is, the free energy of the system, $F$, is given by

$$
F=n_{\mathrm{w}} u_{\mathrm{w}}+n_{\mathrm{s}} u_{\mathrm{s}}+f_{\mathrm{v}}\left(n_{\mathrm{w}}+n_{\mathrm{s}}\right)
$$

where $n_{\mathrm{w}}$ and $n_{\mathrm{s}}$ are the numbers of water and guest molecules, and $u_{\mathrm{w}}$ and $u_{\mathrm{s}}$ are the potential energies of water-water interactions and the sum of water-guest and guest-guest interactions, respectively. The interaction energy is dependent only on density, but the vibrational free energy is a function of both density and temperature. In order to eliminate the effect of the fixed volume on thermodynamic properties and to take the thermal expansion into account, the normal mode analyses are also performed by elongating and shrinking the edges of the basic cell by $1.5 \%$; the cell size parameters, $\xi$, are 1 and 1 \pm 0.015 . The free energy $F$ in eq 2 is minimized with respect to volume at each temperature. In ice Ih, the ratios $a / c$ and $b / c$ are fixed to the constant values and the basic cell is uniformly expanded or shrunk.

The force constant matrix $\mathbf{V}$ is decomposed into submatrices, $\mathbf{V}_{\text {ww }}$ for water-water, $\mathbf{V}_{\mathrm{ws}}, \mathbf{V}_{\mathrm{sw}}$ for water-guest, and $\mathbf{V}_{\mathrm{ss}}$ for guest-guest contributions in the case of the occupied hydrates. As reported previously, ${ }^{4} \mathbf{V}_{\mathrm{sw}}$ and $\mathbf{V}_{\mathrm{ws}}$ play a rather minor role in the free energy calculation. The submatrix $\mathbf{V}_{w w}$ for occupied hydrate is different from that for empty hydrate since elements of the former matrix include the guest-water interaction differentiated twice with respect to the coordinate of a water molecule. The difference in $\mathbf{V}_{w w}$ between empty and occupied hydrates accounts mostly for the discrepancy between the measured dissociation pressure and the predicted one based on the original vdWP theory. ${ }^{4}$

\section{Results and Discussion}

a. Difference in Structural and Energetic Properties. There are several distinct hydrogen bond patterns in the crystalline structures arising from proton-disordered forms of ice and clathrate hydrate I. The pattern is represented by the dihedral angles for a hydrogen-bonded pair, an angle for a bond sequence of $\mathrm{HO}(\mathrm{H}) \mathrm{OH}$ neglecting the central hydrogen, $(\mathrm{H})$, between two oxygen atoms since $\mathrm{O}(\mathrm{H}) \mathrm{O}$ is nearly linear for a strongly hydrogen-bonded pair. Clathrate hydrate I has only an eclipsed form. In contrast, only a staggered form is allowed in cubic ice. Both staggered and eclipsed forms are allowed in ice Ih; the population ratio of the staggered form relative to the eclipsed is exactly 3 . We examine distributions of the dihedral angle for a hydrogen-bonded pair in ice Ih and clathrate hydrate structures generated. In clathrate hydrate I, there are indeed only two distinct dihedral angles; the dihedral angle is either 0 or $2 \pi / 3$. The dihedral angles in ice Ih range from 0 to $\pi$, with an interval of $\pi / 3$; there are four distinct angles. The populations in dihedral angles are given in Figure 1 and Table 1 . For a complete set to classify the conformation for a pair of hydrogenbonded molecules, we must specify a pair of dihedral angles. In the case of clathrate hydrate I, there are only two possible combinations of two dihedral angles, which are $(0,2 \pi / 3)$ and $(2 \pi / 3,2 \pi / 3)$, while there are four patterns in ice Ih; they are $(0,2 \pi / 3),(\pi / 3, \pi / 3),(2 \pi / 3,2 \pi / 3)$ and $(\pi / 3, \pi)$. The number of occurrences for each pattern is also given in Table 1 . The relative occurrence in clathrate hydrate $I$ is $(2: 0: 1: 0)$ and that in ice Ih is $(2: 3: 1: 6)$.

Each hydrogen bond pattern expressed by a combination of dihedral angles has a different interaction energy. For four combinations of dihedral angles given above, the interaction energies in ice Ih are $-21,-19,-26$, and $-24 \mathrm{~kJ} \mathrm{~mol}^{-1}$, respectively. In clathrate hydrate $\mathrm{I}$, the interaction energy of the hydrogen-bonded pair is either -21 or $-26 \mathrm{~kJ} \mathrm{~mol}^{-1}$. These 


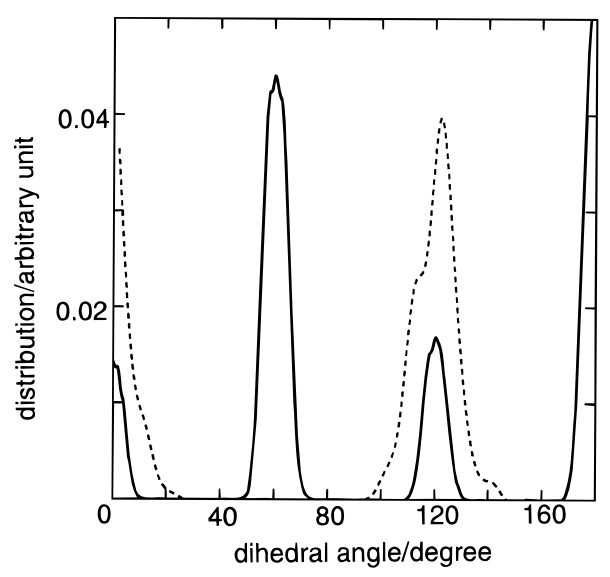

Figure 1. Dihedral angle distribution for individual water molecules in hexagonal ice (solid line) and empty hydrate I (dotted line).

TABLE 1: Percentages of a Single Dihedral Angle (Above) and Combination of Two Dihedral Angles (Below) for a Hydrogen-Bonded Pair for Ice Ih and Clathrate Hydrate I

\begin{tabular}{lrrrr}
\hline & \multicolumn{4}{c}{ angle } \\
\cline { 2 - 5 } & 0 & $\pi / 3$ & $2 \pi / 3$ & $\pi$ \\
\hline Ih & 8 & 50 & 17 & 25 \\
hydrate I & 33 & 0 & 67 & 0 \\
\hline & \multicolumn{5}{c}{ combination of angles } \\
\cline { 2 - 5 } & $(0,2 \pi / 3)$ & $(\pi / 3, \pi / 3)$ & $(2 \pi / 3,2 \pi / 3)$ & $(\pi / 3, \pi)$ \\
& eclipsed & staggered & eclipsed & staggered \\
\hline Ih & 17 & 25 & 8 & 50 \\
hydrate I & 67 & 0 & 33 & 0
\end{tabular}

energy differences must be reflected in a distribution of hydrogen bond energy. The pair interaction energy distributions at $0 \mathrm{~K}$ for ice $\mathrm{Ih}$ and clathrate hydrate I are shown in Figure 2, which are defined as

$$
x_{\mathrm{p}}(v)=\left\langle 1 / N \sum_{i} \sum_{j \neq i}^{N} \delta(v-\phi)\right\rangle
$$

where \langle\rangle indicates the ensemble average and $\phi$ is the TIP4P pair potential function. There are two peaks corresponding to distinct hydrogen bond patterns in clathrate hydrate I. On the other hand, there are three distinct hydrogen bonds in ice Ih; a peak around $-20 \mathrm{~kJ} \mathrm{~mol}^{-1}$ may be further decomposed into two peaks. The peak height at the lowest interaction energy is higher in clathrate hydrate I than in ice Ih. However, the total potential energy for ice Ih is lower than that for clathrate hydrate I because there are a large number of hydrogen bonds of intermediate strength at around $-24 \mathrm{~kJ} \mathrm{~mol}^{-1}$ in ice Ih.

The arrangement of oxygens in clathrate hydrate I is different from that of ice Ih beyond the second nearest neighbors. This is viewed from the radial distribution functions (RDFs). The RDFs at $0 \mathrm{~K}$ are shown in Figure 3. The first peaks corresponding to the hydrogen-bonded pair are omitted since their heights and locations are different from each other only slightly. All the peaks are well separated from each other unlike liquid water. A difference emerges in the location of the third peak, as expected from the difference in the dihedral angle distribution. Although those structures from which RDFs are calculated have no thermal energy, the peaks are rather wide due to the random distribution of protons.

The densities of state of intermolecular vibration for ice Ih and empty clathrate hydrates I averaged over six configurations are shown in Figure 4a. The intermolecular vibrational motions are split completely into translational and rotational motions of

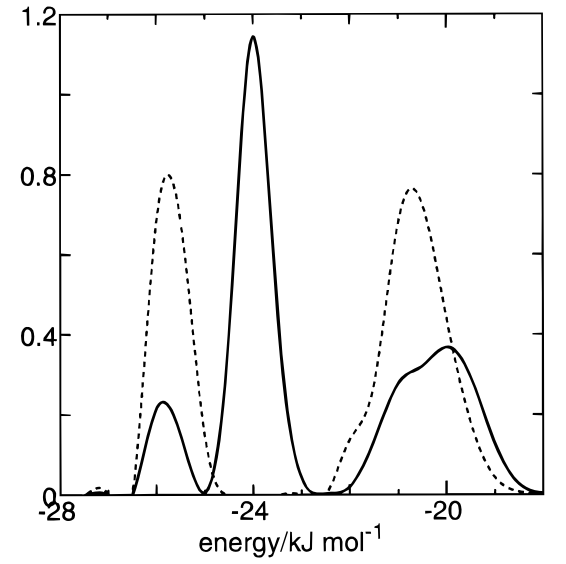

Figure 2. Pair interaction energy distribution for individual water molecules in hexagonal ice (solid line) and empty hydrate I (dotted line).

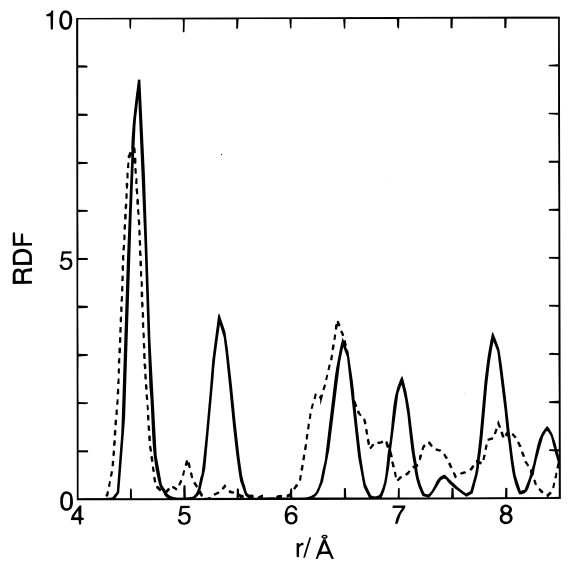

Figure 3. Radial distribution functions for the oxygen pair in hexagonal ice (solid line) and empty hydrate I (dotted line).

individual molecules. The density of state for empty clathrate hydrate I has several peaks at different positions from ice Ih in the lower frequency regions corresponding to the translational motions of the individual molecules. However, the overall patterns are similar to each other. A main difference appears in the higher frequency region composed of rotational motions of individual water molecules: The single peak in the higher frequency region for ice Ih is split into two parts in clathrate hydrate I (both for empty and occupied hydrates).

The density of state calculated from only $\mathbf{V}_{\mathrm{ww}}$ for the occupied hydrate is different from that for the empty hydrate. Since guest molecules have only translational degrees of freedom, the coupling between guest and water is limited to translational motions. The densities of state for empty and occupied hydrate I calculated from only $\mathbf{V}_{\mathrm{ww}}$ are shown in Figure $4 \mathrm{~b}$. Agreement with experiment is semiqualitative. ${ }^{21}$ As expected, occupation of cages gives rise to the blue shift of the peaks in the lower frequency region $\left(0-300 \mathrm{~cm}^{-1}\right)$, which in turn gives higher free energy of cage occupation than that evaluated according to the original vdWP theory.

b. Free Energy and Thermal Expansivity. We calculate the free energy values with three different densities at a given temperature. The intermediate $(\xi=1)$ cell size has, in most of the cases, the lowest free energy among the three in the temperature range we are interested in $(153-273 \mathrm{~K})$. Thus, the minimum free energy is accurately calculated by fitting those three values to a quadratic function against cell volume. The equilibrium volume at a given temperature is calculated together with the free energy. It should be noted that even empty hydrate has a minimum free energy against volume change, which 

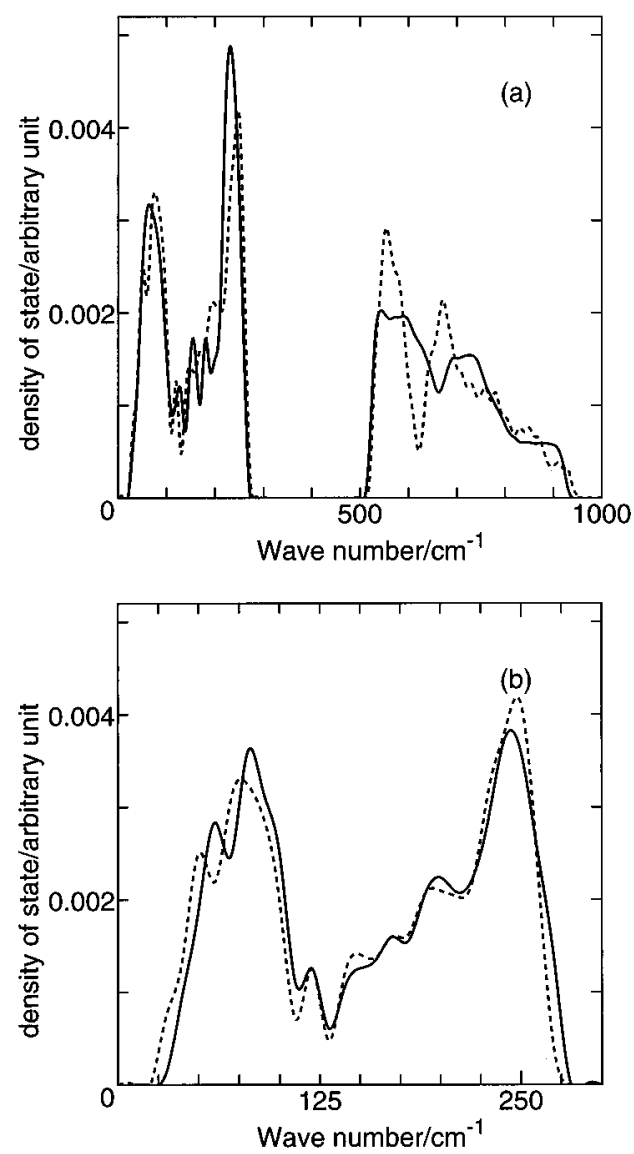

Figure 4. Densities of state for intermolecular vibrational motions (a) for hexagonal ice (solid line) and empty hydrate I (dotted line) and (b) for occupied hydrate I by xenon (solid line) and empty hydrate I (dotted line) as a function of wave number.

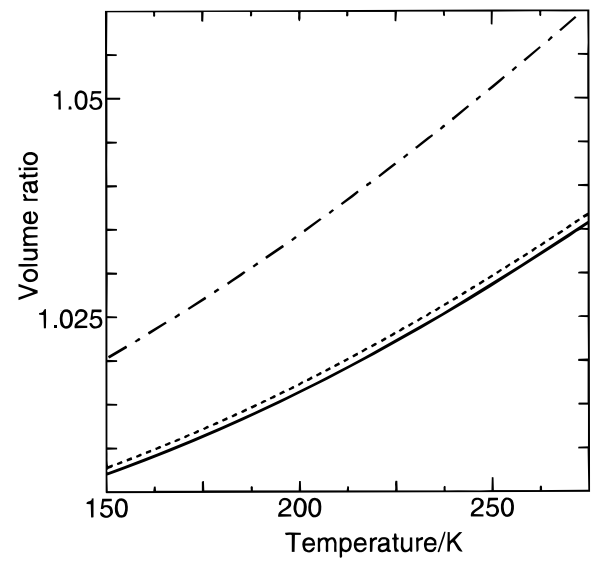

Figure 5. Temperature dependence of volume for hexagonal ice (solid line), empty hydrate I (dotted line), and occupied hydrate I by xenon (dash-dot line). The volumes are scaled so that they are unity at zero temperature.

indicates that empty hydrate could be metastable. The relative volume with reference to the volume at $0 \mathrm{~K}$ is plotted in Figure 5. The volumes for ice Ih, empty hydrate I, and occupied hydrate increase with temperature. The volume for occupied hydrate I increases more abruptly than that for either empty hydrate or ice Ih.

The linear thermal expansivities for ice Ih, empty hydrate I, and occupied hydrate I are shown as a function of temperature in Figure 6. The thermal expansivity for occupied hydrate at $200 \mathrm{~K}$ is $1.04 \times 10^{-4} \mathrm{~K}^{-1}$, which is to be compared with the experimental value, $0.77 \times 10^{-4} \mathrm{~K}^{-1}$ (for ethylene oxide hydrate structure I). ${ }^{16}$ The calculated thermal expansivity for ice Ih,

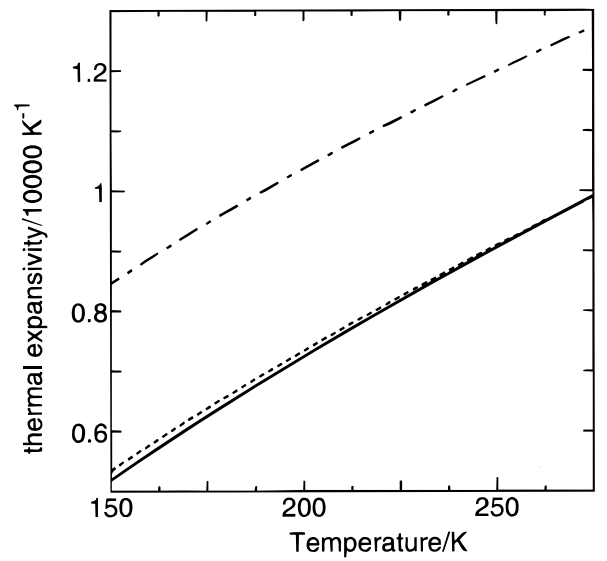

Figure 6. Temperature dependence of thermal expansivity for hexagonal ice (solid line), empty hydrate I (dotted line), and occupied hydrate I by xenon (dash-dot line).

$0.72 \times 10^{-4} \mathrm{~K}^{-1}$, at $200 \mathrm{~K}$ (average over $a, b$, and $c$ axes) is fairly larger than the experimental value, $0.56 \times 10^{-4} \mathrm{~K}^{-1}$. It is interesting that the calculated thermal expansivity for empty hydrate, $0.73 \times 10^{-4} \mathrm{~K}^{-1}$, is almost the same as that for ice Ih. Although a difference in thermal expansivity between experimental and theoretical values is not so small, the calculated value for occupied hydrate is larger than that for ice Ih and empty hydrate. This suggests that our calculation is reliable in examining the origin of the large discrepancy of the thermal expansivity (a part of anharmonicity is taken into account in the potential energy at its minimum structure when the volume is changed to find the free energy minimum).

The large thermal expansivity is originated from the existence of guest molecules since the thermal expansivity of empty hydrate is much smaller than that of occupied hydrate and is almost the same as that of ice. It is important to examine which part of guest contributions plays a major role in increasing the thermal expansivity: (1) vibrational free energy change of the host lattice, (2) potential term of the guest-water interaction, (3) vibrational free energy of guest molecules in the cages. The appropriate, though approximate, free energy of the system, $F_{1}$, is written as

$$
F_{1}=n_{\mathrm{w}}\left(u_{\mathrm{w}}+f_{\mathrm{w}}^{\prime}\right)+n_{\mathrm{s}}\left(u_{\mathrm{s}}+f_{\mathrm{s}}^{\prime}\right)
$$

where $f_{\mathrm{w}}^{\prime}$ and $f_{\mathrm{s}}^{\prime}$ are the harmonic vibrational free energies for water and guest, which are calculated from force constant submatrices $\mathbf{V}_{\mathrm{ww}}$ and $\mathbf{V}_{\mathrm{ss}}$ separately, neglecting $\mathbf{V}_{\mathrm{ws}}$ and $\mathbf{V}_{\mathrm{sw}}$. It is known that the vibrational free energy is well separated into water and guest terms since $\mathbf{V}_{\text {ws }}$ and $\mathbf{V}_{\text {sw }}$ contribute little to the total free energy. ${ }^{4}$ The vibrational free energy difference of water $\left(f_{\mathrm{w}}^{\prime}-f_{\mathrm{w}}^{0}\right)$ is missing in the original vdWP treatment where $f_{\mathrm{w}}^{0}$ is the vibrational free energy of empty hydrate $\mathrm{I}$. This amounts to $0.2 \mathrm{~kJ} \mathrm{~mol}^{-1}$ at $200 \mathrm{~K}$ and may be significant in larger thermal expansivity of occupied hydrate. Therefore, the free energy of only water,

$$
F_{2}=n_{\mathrm{w}}\left(u_{\mathrm{w}}+f_{\mathrm{w}}^{\prime}\right)
$$

is minimized to calculate the thermal expansivity, which is different from the free energy of empty hydrate,

$$
F_{0}=n_{\mathrm{w}}\left(u_{\mathrm{w}}^{0}+f_{\mathrm{w}}^{0}\right)
$$

The thermal expansivity by minimizing $F_{2}$ in eq 5 is plotted in Figure 7 together with those for empty hydrate and occupied hydrate by minimizing $F_{0}$ and $F_{1}$. Since the approximate free energy (eq 4 ) is actually the same as that according to eq 2 , the 


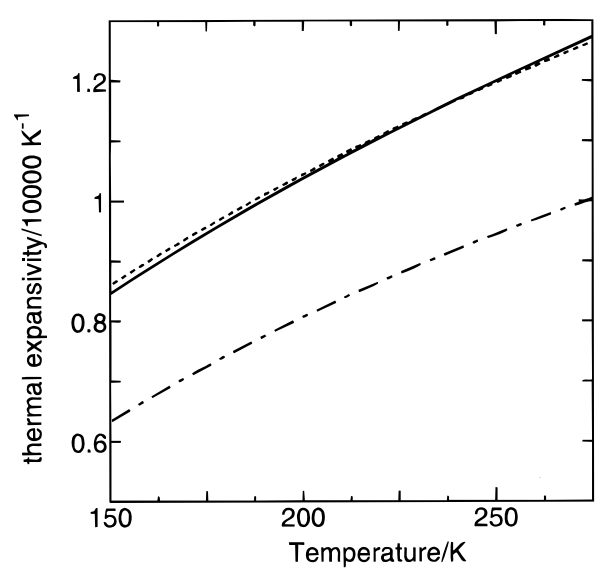

Figure 7. Temperature dependence of thermal expansivities for occupied hydrate by minimizing the free energy in eq 2 (solid line), in eq 4 (dotted line), and in eq 5 (dash-dot line). The free energy in eq 2 takes account of all the matrix elements in diagonalizing the force constant matrix. In eq 4, the submatrices are diagonalized separately. In the free energy according to eq 5, only the submatrix $\mathbf{V}_{\mathrm{ww}}$ is included. See also text.

thermal expansivity is also the same, the former, $1.04 \times 10^{-4}$ $\mathrm{K}^{-1}$, the latter, $1.04 \times 10^{-4} \mathrm{~K}^{-1}$ at $200 \mathrm{~K}$. However, the thermal expansivity from eq $5,0.81 \times 10^{-4} \mathrm{~K}^{-1}$, is only slightly larger than that from eq $6,0.73 \times 10^{-4} \mathrm{~K}^{-1}$, suggesting the guest free energy term $u_{\mathrm{s}}+f_{\mathrm{s}}^{\prime}$ is important to obtain the large thermal expansivity. A further calculation as to which component, $u_{\mathrm{s}}$ or $f_{\mathrm{s}}^{\prime}$, contributes more significantly is also made. The thermal expansivity from eq 4 but dropping the guest interaction energy $u_{\mathrm{s}}$ is $1.22 \times 10^{-4} \mathrm{~K}^{-1}$, which is fairly large compared with that including the guest interaction. Therefore, a main source of the larger thermal expansivity is the vibrational motions of guest molecules inside the cages which dominates over the guest interaction energy $u_{\mathrm{s}}$. The guest interaction term diminishes the large thermal expansion.

The large thermal expansivity is reproduced via the free energy-volume relation. Then, a question is raised as to the guest size dependence of the thermal expansivity: which guest alters the thermal expansivity more seriously, larger or smaller? As has been discussed by Tse et al., a larger guest reduces the free volume of the void space in clathrate hydrates and increases the collision effect with cages. This consequently gives rise to a larger cell size at a given temperature. The thermal expansivity is a further thermal effect on the volume, which is already expanded even at very low temperatures by accommodating a large guest. To examine the size effect, the same procedure is applied to a clathrate hydrate encaging an artificial guest, whose LJ size parameter is $10 \%$ larger $(\zeta=1.1)$ than the original LJ size parameter for xenon $(\zeta=1)$. The equilibrium cell volume at $200 \mathrm{~K}$ is larger by $2 \%$ than that encaging normal xenon $(\xi=$ 1). However, the thermal expansivity, $0.82 \times 10^{-4} \mathrm{~K}^{-1}$, is nearly the same as that of the empty hydrate value.

This implies that the larger guest molecule reduces the anharmonicity which is responsible for the large thermal expansivity. The potential surface of a guest with surrounding water molecules becomes harmonic with an increase in guest size, but the harmonic potential does not contribute to the thermal expansion. The difference in thermal expansivity between two kinds of guests (i.e., $\zeta=1$ and 1.1) becomes smaller with decreasing temperature. An effective potential surface of a guest molecule in a cage becomes quadratic at high temperatures, unlike normal solids. This is examined in detail in conjunction with the anomalous thermal conductivity of clathrate hydrates. ${ }^{14}$ The anharmonicity of guest vibrations, which is the main source of the large thermal expansivity,
TABLE 2: Einstein Frequencies of Empty and Occupied Hydrates (in $\mathbf{c m}^{-1}$ ) and Approximate Griineisen Constant at $200 \mathrm{~K} \gamma_{\mathrm{i}}$, Where $i$ Specifies Mode Character: The Modes Are Classified into Translational (trans), Rotational (rot) Motions of Water and Guest Translations (guest), Which Are Averages of the Diagonal Terms of the Force Constant Matrix; $\zeta$ Is the Scale Factor of the Guest Size with Reference to Xenon; In Parentheses, the Heat Capacity Weighted Griineisen Constant Is Given

\begin{tabular}{llcc}
\hline & \multicolumn{2}{c}{ type } \\
\cline { 2 - 4 } & empty & occupied $(\zeta=1)$ & occupied $(\zeta=1.1)$ \\
\hline \multirow{3}{*}{ Einstein Frequency } \\
trans & 187 & 189 & 193 \\
rot & 682 & 682 & 683 \\
guest & & 24 & 37 \\
& & Grüneisen Constant \\
trans & $2.27(1.87)$ & $2.32(1.64)$ & $2.33(1.65)$ \\
rot & $0.71(0.13)$ & $0.72(0.11)$ & $0.72(0.11)$ \\
guest & & $3.34(0.47)$ & $2.94(0.42)$
\end{tabular}

diminishes at high temperatures. It has been shown experimentally that there is no discernible difference in lattice constant for a large range of hydrates with guest molecules of different sizes. $^{22}$ Anharmonic vibrational motions arising from guest rotational motions will make a large contribution to the thermal expansivity, which are not dealt with in the present study.

We examine which kind of vibrational motion is the key to the large thermal expansivity, by separating vibrations into three terms depending on mode character, host translations, host rotations, and guest translations. To do this, approximate Grüneisen constants averaged over modes belonging to the same mode character are evaluated separately. Instead of normal modes in which three kinds of motions are mixed, it is preferable to classify vibrational modes into three categories by its character as explained above. A trace of the matrix, $\operatorname{tr}\left(\mathbf{m}^{-1 / 2}\right.$ $\left.\mathbf{V} \mathbf{m}^{-1 / 2}\right)$, is invariant under unitary transformation, and the square root of an average of diagonal elements in each molecule gives its Einstein frequency. In the same sense, the square root of an average in each mode character gives an approximate Einstein frequency specific to each character of mode. Those Einstein modes are calculated by diagonal elements of the massweighted force constant matrices: The diagonal terms are averaged separately, depending on mode character; translational and rotational degrees of freedom for water and translational degree of freedom for guest. Each frequency is listed in Table 2. Although the translational frequency is affected little in Xe with $\zeta=1$, both frequencies of water and guest translational motions are shifted to the higher side in the case of $\zeta=1.1$. The shifts are remarkably larger. In contrast, rotational motions of water are not affected. The Grüneisen constants calculated at $200 \mathrm{~K}$ are also given in Table 2, which agrees reasonably with that by experimental work ${ }^{23}$ (it is approximately unity over a wide range of temperatures). The Grüneisen constant from guest vibrations becomes smaller for larger guests. This is again consistent with the results obtained in the last paragraph.

\section{Concluding Remarks}

The thermal expansivity of xenon clathrate hydrate has been calculated by evaluating the free energies at various temperatures in order to explain the origin of the large thermal expansivity of clathrate hydrates compared with that of ice. Several protondisordered configurations for hexagonal ice and type I clathrate hydrates are generated. The free energy of the system is approximated to the minimum potential energy, the harmonic free energy and the configurational entropy arising from the disordered nature of protons. The free energy at a given temperature is minimized with respect to the volume of the 
system. Thus, the equilibrium volume is obtained. This provides an alternative way to evaluate the thermal expansivity from only intermolecular interaction potentials.

The experimental observation that the larger thermal expansivity of clathrate hydrates than ice is successfully reproduced. It is found that the large expansivity of clathrate (structure I) arises from the existence of guest molecules and that a difference in oxygen arrangement between clathrate hydrates and ice plays a minor role. An effective potential surface of a smaller guest is more anharmonic and therefore causes larger thermal expansivity. Raising temperature has the same effect as increasing the guest size and diminishes the expansivity relative to ice.

It should be noted that rotational motion of an aspherical guest molecule, which is not treated in the present study, may contribute to the anharmonic free energy, and therefore a further difference from ice is expected in clathrate hydrates such as ethylene oxide hydrate. A further study is required to examine the effect of rotational motions of guests on thermal expansivity and also conductivity.

Acknowledgment. The authors thank K. Kiyohara and I. Okabe for providing proton-disordered ice Ih and clathrate hydrate I structures. This work is supported partially by a Grantin-Aid from the Ministry of Education, Science and Culture. Most of the calculations were carried out with the use of supercomputers at the Institute for Molecular Science.

\section{References and Notes}

(1) Davidson, D. W. Water-A Comprehensive Treatise Franks, F., Ed.; Plenum: New York, 1973; Vol. 2.

(2) Sloan, E. D. Clathrate Hydrate of Natural Gases; Marcel-Dekker: New York, 1990.
(3) van der Waals, J. H.; Platteeuw, J. C. Adv. Chem. Phys. 1959, 2,

(4) Tanaka, H.; Kiyohara, K. J. Chem. Phys. 1993, 98, 4098.

(5) Tanaka, H.; Kiyohara; K. J. Chem. Phys. 1993, 98, 8110.

(6) Tanaka, H. J. Chem. Phys. 1994, 101, 10833. 1423.

(7) Andersson, P.; Ross, R. G. J. Phys. C: Solid State Phys. 1983, 16,

(8) Ashworth, T.; Johnson, L. R.; Lai, L. High Temp.-High Press. 1985, 17, 413.

(9) Handa, Y. P.; Cook, J. G. J. Chem. Phys. 1987, 91, 6327.

(10) Tse, J. S.; White, M. A. J. Phys. Chem. 1988, 92, 5006.

(11) Tse, J. S.; Klein, M. L.; McDonald, I. R. J. Chem. Phys. 1983, 78, 2096.

(12) Tse, J. S.; Klein, M. L.; McDonald, I. R. J. Chem. Phys. 1984, 81, 6146.

(13) Tse, J. S. J. Incl. Phenomena 1994, 17, 259.

(14) Inoue, R.; Tanaka, H.; Nakanishi, K. J. Chem. Phys. 1996, 104, 9568.

(15) Roberts, R. S.; Andrikidis, C.; Tanish, R. J.; White, G. K. Proceedings of the 10th International Cryogenics Engineering Conference; Callan, H., Bergland, P., Krusius, M., Eds; Butterworths: Helsinki, 1984

(16) Tse, J. S.; McKinnon, W. R.; Marchi, M. J. Phys. Chem. 1987, $91,4188$.

(17) Touloukian, Y. S.; Kirby, R. K.; Taylor, R. E. Lee, J. Y. R. Thermophysical Properties of Matter; Plenum: New York, 1977; Vol. 13, p 263.

(18) Jorgensen, W. L.; Chandrasekhar, J.; Madura, J. D.; Impey, R. W.; Klein, M. L. J. Chem. Phys. 1983, 79, 926.

(19) Hirschfelder, J. O.; Curtiss, C. F.; Bird, R. B. Molecular Theory of Gases and Liquids; Wiley: New York, 1954.

(20) Eisenberg, D.; Kauzmann, W. The Structure and Properties of Water; Oxford University Press: London, 1969.

(21) Tse, J. S. Chem. Phys. Lett. 1993, 215, 383.

(22) Davidson, D. W.; Handa, Y. P.; Ratcliffe, C. I.; Ripmeester, J. A.; Tse, J. S.; Dahn, J. R.; Lee, F.; Calvert, L. D. Mol. Cryst. Liq. Cryst. 1986, 141, 141.

(23) Tse, J. S. J. Phys. C 1987, 1, 543. 